\title{
CONE)-(oES
}

CIÊNCIA E TECNOLOGIA

\section{INTERNACIONALIZAÇÃO, EDUCAÇÃO PROFISSIONAL, ARTE E CULTURA: UMA REFLEXÃO.}

\author{
AnA Judith ZEPEDA ORTEGA ${ }^{1}$ \\ ${ }^{1}$ Universidad de Valparaíso \\ <ana.zepeda.o@gmail.com> \\ DOI: <https://doi.org/10.21439/conexoes.v14i5.1772>
}

\begin{abstract}
Resumo. A internacionalização do ensino superior tem mostrado mudanças nas últimas décadas, em conjunto com as transformações socioculturais e econômicas que as nações experimentaram. Isso obrigou as Instituições de Ensino Superior a rever suas ações na perspectiva desses ventos de mudança. Por outro lado, as expressões culturais e artísticas sempre foram diversificadas e enriquecidas pela circulação de seus expoentes, entre diferentes espaços territoriais, delimitados ou não por uma linha de fronteira geográfica. No Ensino Superior, as artes e a cultura residem em vários espaços do trabalho dessas instituições, dentro das quais são contadas: a formação de profissionais; ensinar através das artes; as expressões artísticas e culturais da comunidade educativa e; a custódia e cuidado do patrimônio cultural. Em cada uma dessas áreas elas desempenham um papel importante, no entanto, não foi suficientemente analisado qual seria o seu lugar em relação à internacionalização da Educação Superior. Portanto, o objetivo deste trabalho foi examinar as esferas do ensino superior, onde artes e cultura podem se tornar parte do processo de internacionalização do ensino superior. Este estudo foi realizado com base em uma revisão documental, focada na busca de elementos que pudessem contribuir para uma análise crítica e reflexiva sob diferentes ângulos.
\end{abstract}

Palavras-chaves: Internacionalização. Ensino superior. Arte. Cultura.

\section{INTERNATIONALIZATION OF HIGHER EDUCATION, ART AND CULTURE: A REFLECTION}

\begin{abstract}
The internationalization of higher education has shown changes in recent decades, in conjunction with the socio-cultural and economic transformations that nations have experienced. This has forced higher education institutions to review their actions in the perspective of these winds of change. On the other hand, cultural and artistic expressions have always been diversified and enriched by the circulation of their exponents, between different territorial spaces, delimited or not by a geographical border line. In higher education, arts and culture reside in various spaces of the work of these institutions, within which they are counted: the training of professionals; teaching through the arts; the artistic and cultural expressions of the educational community and; the custody and care of cultural heritage. In all of these areas they play an important role, however, it has not been sufficiently analyzed what would be their place in relation to internationalization of higher education. Therefore, the objective of this work was to examine the spheres of higher education where arts and culture can become part of the process of internationalization of higher education. This study was conducted based on a documentary review, focused on the search for the elements that could contribute to a critical and reflective analysis from different angles.
\end{abstract}

Keywords: Internationalization. Higher education. Art. Culture. 


\section{INTRODUÇÃO}

Las expresiones culturales y artística latinoamericana desde sus orígenes han sido diversificadas y enriquecidas por el ir y venir de artistas y continuo flujo de peregrinos, entre los diferentes espacios territoriales, delimitados o no por una línea de frontera geográfica. Teniendo en cuenta, además, que el pasado colonial le ha impreso a las artes y letras latinoamericanas, elementos procedentes de la cultura hispánica o lusa (GUTIÉRREZ, 2010).

Por su parte, el proceso de difusión o propagación que cruza las fronteras de las naciones, no es un fenómeno nuevo en lo que a las artes y la cultura competen. Así es como, en todos los paises de la región se pueden encontrar ejemplos de artistas que se trasladaron a otras naciones para afinar sus destrezas artísticas y por ende también, en ese acto se produjo el intercambio cultural correspondiente. Esto porque, ese artista lleva consigo los rasgos distintivos, espirituales y afectivos que caracterizan a la sociedad o al grupo social al cual pertenece. Todo lo cual quedará expuesto a la construcción de un renovado tejido cultural, producto de la mezcla de elementos culturales de origen con los del lugar de asentamiento (MARÍN, 2007).

En tanto que, la profesionalización de la actividad artistica, particularmente en el contexto latinoamericano, ha sido un proceso paulatino, el cual tuvo lugar en el transcurso del siglo XX (AGUIRRE, 2009). En primera instancia, se fundaron las escuelas de arte, teatro o conservatorio de música, entre otros. Luego estas academias fueron integradas a las universidades. Tal es el caso de la "Escola de Arte Dramática" en Brasil, que se funda en la década del 1940 y luego en los 70 pasa a formar parte de la Universidad de São Paulo (Noya, 1994). El mismo proceso se pudo evidenciar en Chile cuando la Facultad de Bellas Artes de la Universidad de Chile se forma mediante la reunión de la Escuela de Bellas Artes y el Conservatorio Nacional de Música (??). En Argentina, la Escuela Nacional de Bellas Artes Prilidiano Pueyrredón pasó a integrar el Instituto Universitario Nacional del Arte, el cual forma parte del Departamento de Artes Visuales de la Universidad Nacional de las Artes (UNIVERSIDAD NACIONAL DE LAS ARTES 2019; MUÑOZ 2000). Y así, se podrían mencionar muchos ejemplos similares en todo Latinoamérica.

Por otra parte, asistimos a una época de expansión e intercambio entre las naciones, en cuanto a tecnología, conocimientos, bienes y servicios, entre otros. Esto ha abierto los espacios y canales de comunicación entre los paises, los cuales han iniciado un estilo de cooperación internacional, el cual incluye la integración en diversos ámbitos, dentro de los que se encuentra la Educación Superior (ES). Es así como en la primera Declaración de la Conferencia Regional de la Educación Superior en América Latina y el Caribe (CRES) se decía que "... las instituciones de educación superior deben asumir el estudio de la integración latinoamericana...”, al mismo tiempo que recomendaba formular un plan integral de desarrollo que “...posibilite el desarrollo de experiencias mutuas sur-sur y norte-sur...” con el fin de generar un impacto que favorezca la calidad de la enseñanza, apoye y promueva la formación de las redes subregionales, que priorice en área fundamentales, tales como: problemas sociales, medio ambiente, formación de capital humado de alto nivel, el cual pueda liderar los procesos de transformación que requieren los paises (CRES, 1996).

Lo anterior se profundizó en la siguiente reunión de la CRES, en 2008, donde se dijo que "es básico para alcanzar niveles superiores que apunten a aspectos fundamentales de la integración regional”, puesto que, la disponibilidad de recursos humanos altamente capacitados serán fundamentales para que la región pueda enfrentar en mejores condiciones los problemas apremiantes del mundo en general (CRES, 2009) . Finalmente, en la última CRES insta a realizar cambios en los sistemas educativos de manera que se construya una cooperación solidaria de saberes y de recursos entre los países y las instituciones de la región. Además, afirma que la internacionalización es una herramienta fundamental como "para transformar la educación superior, fortalecer sus comunidades y promover la calidad y pertinencia de la docencia, la investigación y la extensión" y que, además, debe ser llevada a cabo sobre la base de una relación solidaria entre iguales (CRES, 2018).

Lo anterior pone en evidencia la progresiva incorporación de las artes y la cultura en la ES, pero también el renovado interés por las cuestiones socioculturales y de integración Latinoamericana. Además, es importante agregar que, en la ES, las artes y la cultura residen en varios espacios del quehacer de dichas instituciones, dentro de los que se cuentan: la formación de profesionales; la enseñanza a través de las artes; las expresiones artísticas y culturales de la comunidad educativa y; la custodia y cuidado del patrimonio cultural. En cada una de estas áreas cumplen un importante rol, sin embargo, no se ha analizado suficientemente cual sería el lugar que le corresponde en lo relativo a internacionalización de la Educación Superior. Por tanto, el objetivo de este trabajo fue examinar las esferas de la Educación Superior donde las artes y la cultura pueden hacerse parte del proceso de internacionalización de la Educación Superior. 
Este estudio se realizó sobre la base de una revisión documental, centrada en la búsqueda de los elementos que pudieran contribuir a un análisis crítico y reflexivo desde diferentes ángulos. Para ello, fue necesario revisar algunos aspectos que facilitarán la comprensión y desarrollo del tema. Estos son: el contexto en el que se llevaría a cabo y los conceptos involucrados; la presencia de las artes y la cultura dentro de la ES y; el horizonte que podría tener la internacionalización del arte y la cultura en ES, teniendo presente los espacios que ellas ocupan.

\section{El Contexto y conceptualización relevante}

En primer lugar, resulta importante revisar el conjunto de condiciones actuales, que son el telón de fondo, sobre el cual se está produciendo el proceso internacionalización de la educación superior. Esto nos permitirá una mejor comprensión de las circunstancias en la cuales se han de desarrollar las manifestaciones artisticas y culturales que involucra la internacionalización de estas.

\subsection{La globalización}

Lo primero que es importante de analizar es lo relativo a "la globalización". Este no es un concepto nuevo en el ámbito de la economía, pero, en las últimas décadas se ha desarrollado y profundizado. Este fenómeno, desde una perspectiva económica, se ha dado básicamente porque el dinero, la mercadería, la producción y otros bienes, han circulado más allá de las fronteras de las naciones, a una escala sin precedentes. Esto porque, los medios de producción y la fuerza laboral se convirtieron en transnacionales. A la par, se ha expandido el comercio internacional, aun cuando en muchos paises se ha intentado proteger su producción nacional imponiendo medidas de gravamen para las importaciones. Pero también el flujo del dinero ha sido a escala mundial, ya sea con el fin de obtener mayores ganancias en paises con menores cargas impositivas o bien mediante la inversión en bienes de infraestructura que aseguren la cadena de producción o extracción de la materia prima. Sumado a ello, el proceso productivo se ha llevado a lugares donde la mano de obra es menor costo (FANJUL 2018).

En simultaneo, a lo antes descrito, se han sentado las bases para un proceso mucho más amplio de integración mundial que traspasa lo político, social, tecnológico, académico y cultural, entre otros. Las fronteras se desdibujan y gracias a los avances de las comunicaciones, las noticias se propagan a gran velocidad y en breve tiempo, o bien, son transmitidas en tiempo real
(FERNÁNDEZ, 2002). La percepción de un mundo más interconectado ha llevado a pensar en el planeta como una "aldea global". Sin embargo, hay voces que ponen en duda tal concepto, pues en una aldea se da un grado de conocimiento, intimidad y solidaridad entre sus habitantes, lo cual está muy distante de lo que vivimos hoy en día, donde el avance de las tecnologías ha propiciado un contacto despersonalizado y muchas veces indiferente por lo que le acontece al otro (FAZIO. 2018).

\subsection{Los avances de las tecnologías comunicacio- nales}

Por otra parte, el nuevo escenario que plantea el explosivo avance de las tecnologías interactivas es otra de las circunstancias que se debe tener en consideración, particularmente lo relacionado con los medios de comunicación. Esto porque han proporcionado los instrumentos como para que el ciudadano común acceda a múltiples posibilidades de información y servicios de toda índole. Además, le ha permitido generar redes de comunicación y espacios virtuales de encuentro con personas de las más variadas caracteristicas y lugares geográficos (DEHESA; KRUGMAN, 2007).

La globalización y los cambios impulsados por los avances tecnológicos de las comunicaciones han socavado las estructuras sociales y culturales, tensionando las relaciones entre los grupos sociales, generando variadas reacciones, las que van desde la aceptación de lo nuevo sin cuestionamiento, hasta la reivindicación de lo propio con el consiguiente rechazo de lo foráneo.

\subsection{La cultura y los cambios socioculturales}

Ahora bien, si tomamos como definición de cultura la dada por la Organización de las Naciones Unidas para la Educación, la Ciencia y la Cultura (UNESCO), donde señala que corresponde "al conjunto de los rasgos distintivos, espirituales y materiales, intelectuales y afectivos que caracterizan a una sociedad o un grupo social" (UNESCO, 2001), cuando se produce la aceptación de lo foráneo conlleva la pérdida los valores distintivos de los pueblos, conduciéndolos a la homogeneidad cultural. Esto ha sido fuente de críticas, donde se señala que la globalización y las nuevas tecnologías se han constituido en una nueva forma de imperialismo cultural, tal como ya ocurrió en Latinoamérica, donde los colonizadores españoles y portugueses impusieron su idioma, sus costumbres, su religión, sus sistemas de gobierno, economía y construcción urbana, entre otros, sometiendo y despojando a nuestros pueblos originarios de su patrimonio económico y cultural (FIGUEROA, 2015. 
SOLIS, 2005). Es por lo que se argumenta que, ésta forma de confrontación cultural, amenaza la identidad cultural de las sociedades involucradas, pues tiende a favorecer la idea de cultura global, donde la cultura de las grandes potencias se impondrá sobre las naciones menos poderosas (BOLÍVAR et al. 2001).

Por otro lado, hay quienes ven en estos procesos, una oportunidad de convivencia multicultural, de enriquecimiento mutuo y de acatamiento de las diferencias (KAUFMANN, 2011). Esto requerirá una reevaluación de la manera en que se ha de conducir la sociedad. En esta posición, hay quienes hablan de la "hibridación" de la cultura, lo cual sería “... un proceso de interacción entre lo local y lo global, lo hegemónico y lo subalterno, el centro y la periferia... ", todo lo cual conduce a "... un proceso de transacciones culturales que pone de manifiesto como las culturas globales son asimiladas localmente..." (NILAN; FEIXA, 2010).

Sin embargo, la secuencia de los hechos que conllevan estos fenómenos está en construcción, donde no es fácil predecir la forma en que evolucionarán, ni menos aún sus resultados. Pero, lo que sí está claro, es que para que esto fluya se deben tender los puentes para la convivencia solidaria, en un ambiente de respeto y de tolerancia.

\subsection{La cultura y las manifestaciones artísticas}

Así mismo, la cultura de un pueblo se puede aprehender a través de sus manifestaciones artisticas, por tanto, el arte está íntimamente relacionado con la cultura. El arte se corresponde con las manifestaciones de la actividad humana, donde se interpreta la realidad, vista o percibida por el artista o los artistas, quien o quienes lo dejan plasmado, haciendo uso de algún recurso plásticos, lingüístico, sonoros, visual u otros (Real Academia Española, 2018, CONCEPTO, 2019). Por tanto, a través del arte podemos tomar conocimiento sobre los sistemas de vida, los valores, las tradiciones y las creencias de una sociedad. Es decir, podemos conocer "los rasgos distintivos, espirituales y materiales, intelectuales y afectivos que caracterizan a una sociedad o un grupo social", lo que en definitiva es la cultura (UNESCO. 2001). Así es como, a través del arte, los seres humanos podemos reflexionar sobre nosotros mismos, sobre nuestro entorno y sobre el significado que le damos a los elementos culturales propios y ajenos.

Por otra parte, los avances tecnológicos han hecho también que los procesos creativos hayan cambiado, ampliando sus horizontes en cuanto las formas de expresión artística, diversificando los espacios, la manera de producción, etc. A la par, ha cambiado también la forma de exhibir el arte, donde hoy en día, la concep- ción de una muestra confinada (museos, teatros, cines, etc.) ha dado paso a la muestra itinerante, dinámica e interactiva, llegando incluso a intervenir los espacios urbano, donde por ejemplo se ha podido ver que un coro surge de entre los pasajeros de una estación de trenes, cantando villancicos (ELEKTRART, 2019). Estas nuevas formas de exposición artisticas y el uso de las nuevas tecnologías ha permitido que el arte llegue a más personas, pero también ha abierto la puerta como para que se puedan experimentar con nuevas técnicas, hacer uso de nuevos recursos, ampliando las formas de creación y de transmisión emociones (GIANNETTI, 2002. REDACCIóN, 2018).

\subsection{El patrimonio cultural}

El patrimonio cultural, según la UNESCO es “nuestro legado del pasado, con lo que vivimos hoy y lo que transmitimos a las generaciones futuras. Nuestro patrimonio cultural y natural son fuentes insustituibles de vida e inspiración" (UNESCO, 2019). Sin embargo, hay quienes lo consideran como un espacio de imaginación, puesto que para los seres humanos cualquiera de sus producciones, pueden adquirir un valor de interés patrimonial, donde el reto está en “... reconocerlos y transformarlos en espacios de diálogo, de negociación, una oportunidad para construir realidades..." (MECA, 2016). Empero, la noción de bien cultural ha evolucionado, donde actualmente incluyen otros elementos, tales como los: folklóricos, bibliográficos, documentales, fenómenos de la naturaleza superlativos o áreas de excepcional belleza (LLULL, 2005).

Así es como, el patrimonio cultural se constituye en un elemento importante en los procesos de enseñanza y aprendizaje, puesto que la valoración de estos bienes son parte de la construcción de la identidad y, por ende, también están sujetos a la circulación internacional. Por tanto, se ven enfrentadas a las mismas tenciones revisadas anteriormente, en razón a las artes, la cultura y las trasformaciones sociales a las que asistimos hoy.

\subsection{La educación superior más allá de las fronteras}

La educación superior no ha sido inmune a los vientos de cambio que ha traído la globalización y el avance de las tecnologías. Como consecuencia de ello, las instituciones de educación superior y los gobiernos han impulsado una variedad de acciones tendientes a materializar la apertura y circulación de la educación superior más allá de las fronteras (HRISTOVA; PETROVSKA; DIMITROVA, 2013).

Sin embargo, tal como se mencionó en la introducción, este no es un fenómeno nuevo, sino que se ha 
transformado, tanto en su conceptualización como en la forma en que se ha llevado a cabo por parte de las diferentes Instituciones de Educación Superior (IES). Es así como antiguamente, la internacionalización era entendida como la cooperación internacional universitaria, con claro énfasis en: la movilidad de estudiantes y docentes; la organización de eventos en conjuntos con IES de otros paises (preferentemente del hemisferio norte) y, la generación de vínculos entre IES que las fortaleciera y proyectara internacionalmente. En cambio, a fínales de los años ochenta, el concepto de internacionalización experimenta importantes cambios, lo cual amplia los espacios y referentes del concepto, lo que aún se encuentra en construcción. Este nuevo concepto incluye las actividades ya mencionadas, pero pone el foco en el papel que le cabe a la IES y no en la relación entre IES. Además, "implica desarrollar un proceso de incorporación de lo internacional en las acciones” que la IES realiza (BENEITONE, 2014), es decir la integración de lo "internacional e intercultural a las funciones de enseñanza, investigación y servicio de la institución" (KNIGHT, 1994).

Por tanto, la internacionalización, desde este nuevo enfoque, ha aumentado y diversificado las actividades y formas en que se implementa en las IES. Esto ha significado que en muchos casos se haya incorporado al proceso de enseñanza y aprendizaje. Es así como se ha propiciado la enseñanza internacional, la educación multi e intercultural, incorporación del estudio de lenguas extranjeras en los programas de estudios e internacionalización del currículo. Y con relación a la movilidad, no solo se ha concretado con los miembros de la comunidad académica que habitualmente accedían (estudiantes, académicos, científicos), sino que también se han incorporado los gestores y personal administrativo, los cuales han podido acceder a estudios en el extranjero, intercambio de experiencias con personas que realizan actividades similares en otros paises, etc.. Así mismo, el intercambio habitual se ha visto enriquecido con el acceso a instalaciones y tecnología avanzadas y capital humano disponible en lugares remotos. Pero también, con la formación de alianzas y redes transfronterizas, estableciendo acuerdos bilaterales o multilaterales, entre otros (WIT; GACEL-ÁVILA; KNOBEL, 2017).

En conjunto con lo anterior, también, se han instalado sedes de universidades extranjeras, por ejemplo, la apertura de una sede de la Universidad de Bolognia en Buenos Aires. También, han proliferado los programas de educación a distancia, de doble titulación o con títulos extranjeros. Se suma a esto, el traslado de uno a más docente de universidades extranjeras, particularmente de paises desarrollados, hacia instituciones de educa- ción superior de otras regiones como por ejemplo a Latinoamérica (CRAVINO; ROLDÁN, 2018: ALTBACH; KNIGHT, 2006).

\subsection{La influencia del Plan Bolonia en la ES de Ame- rica Latina}

La Declaración de Bolonia surge de la reunión de Ministros de Educación provenientes de paises europeos, realizada en Bolonia en 1999. En esta se sientan las bases para establecer un Espacio Europeo de Educación Superior (EEES), cuya finalidad de promover un sistema de educación superior europeo que sea convergente y compatible, de manera tal que facilite la empleabilidad, la movilidad y el reconocimiento de los títulos universitarios en toda Europa. Además, apunta al establecimiento de un sistema de créditos que facilite la movilidad de estudiantes, eliminando también los obstáculos para que se pueda producir la libre circulación de los estudiantes entre la IES europeas (EEES, 1999).

El plan Bolonia ha sido fuente de críticas, pues se dice que ha puesto a la IES al servicio de las necesidades del mercado laboral, traicionando el verdadero espíritu fundacional de la Universidades, donde se ha cambiado el concepto de "formación" por el de "instrucción" (Olímpio Bento, 2016, BIANCHETTI, 2014).

En América Latina, es un tema de debate, aun cuando muchas IES han hecho importantes modificaciones a su quehacer, basado en los lineamientos de la declaración de Bolonia, ya sea por iniciativa de las propias IES o por imposición de acuerdos internacionales. Esto, según algunos autores, vulnera la autonomía de la universidad, pues le impone una forma de funcionamiento que no respeta la autodeterminación de su comunidad académica (BIANCHETTI; MAGALHÃES, 2015). Teniendo en cuenta que las decisiones participativas al interior de la comunidad educativa de las IES fue uno de los puntos centrales del levantamiento estudiantil de la Universidad Nacional de Córdoba, Argentina, en 1918, lo cual ha sido fuente de inspiración para las IES de Latinoamérica y por ende altamente valorado en esta región (Las Heras, 2009).

A pesar de que, la implementación del plan Bolonia en Latinoamerica, puede traer consigo oportunidades para avanzar en la implementación de una ES con mayores estándares de calidad y con títulos acreditable en diferentes paises de la región, no es inocua. Esto porque ha puesto en tensión lo relativo a los conceptos de universidad y sobre qué tipo de profesionales se desea formar en muestra IES. Al respecto se puede decir que, si bien, la empleabilidad es un tema sensible para los egresados de las IES, la formación integral de profesionales y técnicos que, puedan enfrentar los desafíos 
de la sociedad, en el contexto cultural al cual pertenece, puede cobrar similar importancia. Además, es importante tener en cuenta que Latinoamérica, es una región muy diversa, cultural, económica y socialmente, por lo que coexiste una importante asimetría entre las IES de la región, las que conviven con la falta de recursos y las desigualdades sociales (BRUNNER, 2008).

Sin embargo, hay quienes consideran que las objeciones son infundadas puesto que la aplicación del plan Bolonia ha promovido que la ES busquen puntos de acuerdo en un marco de referencia común. También consideran que ha traído la innovación y modernización propia de la educación europea y que la diversidad y diferenciación presente en Latinoamerica no son un obstáculo para lograr la integración regional (GACELÁVILA 2011). Al respecto cabría peguntarse: ¿Por qué lo "moderno e innovador" proveniente de Europa es tan bueno para nuestra región?, ¿por qué la integración latinoamericana ha de hacerse a imagen y semejanza de Europa? y, ¿dónde quedan relegadas nuestras propias iniciativas de integración o de cooperación latinoamerica acorde a nuestra realidad y necesidades?. De ahí que, haya quienes plantean que la integración de la ES latinoamericana debe hacerse desde la solidaridad académica, donde se busque la forma de intercambio latinoamericano teniendo presente “...nuestros problemas y necesidades, desde el sentir de nuestra propia tierra”, tomando en cuenta nuestro patrimonio cultural y nuestras tradiciones. Pero, también, teniendo presente los que no están pudiendo acceder a la ES, debido a las situaciones de pobreza e inequidad social (TELLO 2015).

Entonces, sin desconocer los aspectos positivos que son rescatable del plan Bolonia, es importante considerar que "la producción en serie" de profesionales y técnicos para un mercado laboral globalizado, puede que no sea la mejor estrategia para nuestra región, donde a lo menos se debe tomar en cuenta la creatividad, diversidad y especificidad que tienen las naciones o plurinaciones que componen la región.

\subsection{El arte y la cultura en Declaraciones sobre Edu- cación}

En este acápite se revisa lo relacionado con las artes y la cultura, en algunas declaraciones sobre educación. Esto porque en ellas se puede visualizar la orientación de las recomendaciones, así como también la evolución del contexto en el cual estas se produjeron. Conviene subrayar entonces que en la CRES de1996, se habla del valor de la identidad cultural, donde se dice que la ES se constituye en un elemento importante como para el fortalecimiento de la identidad cultural. Es por lo que las IES deberían velar por "la preservación y el fortalecimiento de la identidad cultural" de la región, para no poner en riesgo "los valores culturales propios de la América Latina y el Caribe” (CRES, 1996). Asimismo, se habla de la necesidad de un entendimiento intercultural, lo cual va en sintonía con los procesos de resistencia política y cultural de los pueblos indígena en la década de los 90. Sin embargo, en ninguno de sus acápites se menciona algo relacionado con las artes.

Luego, en la siguiente década, en la declaración de la CRES 2008, se vuelve a mencionar la importancia del fortalecimiento de la identidad cultural y se explicita que la región es pluricultural y multilingüe, por lo que se debe "... promover la diversidad cultural y la interculturalidad en condiciones equitativas y mutuamente respetuosas ...". Al mismo tiempo, se dice que se debe propiciar el respeto y la defensa de nuestros patrimonios culturales y ambientales (CRES, 2008). Aunque de manera colateral, en esta declaración se menciona, dentro de los retos de oportunidad de la ES, lo que dice relación con que se debe “...tomar en cuenta la riqueza de la historia, de las culturas, las literaturas y las artes..." de la región.

Sin embargo, previo a la CRES 2008, hubo dos iniciativas específicas sobre educación artística que son importante a considerar. La primera de ellas fue la Conferencia Regional de América Latina sobre Educación Artística, realizada en Bogotá, Colombia, en 2005. En ella se dice que se ha observado que los paises de la región "cuentan con un rico patrimonio natural y cultural”, el cual "está siendo amenazado por múltiples y complejos cambios socioculturales y ambientales" que están ocurriendo. Pero, además, en uno de sus considerandos, se señala que "las políticas educativas le dan escasa valoración a la educación artística”, lo cual se manifiesta "en el aislamiento y la desvalorización de esta área del conocimiento” (UNESCO, 2005).

La segunda iniciativa interesante de examinar es la Conferencia Mundial sobre la Educación Artística, realizada en Lisboa, en marzo de 2006, cuyo lema fue: "Construir capacidades creativas para el siglo XXI". En esta se estableció la llamada "Hoja de ruta para la educación artística", con el fin de que se tome conciencia acerca de la importancia que puede tener la educación artística en alcanzar una educación de mejor calidad. En la declaración, se pone de manifiesto que:

\footnotetext{
"El conocimiento y la sensibilización acerca de las prácticas culturales y las formas de arte refuerza las identidades y los valores personales y colectivos, y ayuda a preservar y fomentar la diversidad cultural. La educación artística fomenta tanto la conciencia cultural como las prácticas culturales, y constituye el medio a través del cual el conocimiento y el aprecio por las artes y la cul-
} 
tura se transmiten de una generación a otra." (UNESCO 2006).

Ahora bien, si se tiene en cuenta las dos conferencias sobre educación artística, relativamente contemporáneas a la realización de la CRES 2008, resulta llamativo que, en la declaración de esta última, las artes sean mencionadas de manera colateral y no se profundice en lo que dice relación con la ES de las artes, ni en el rol que estas pueden cumplir en el marco de la educación.

Siguiendo con el análisis, en la última CRES, realizada en Córdoba, Argentina, en 2018, se pudo observar que lo relacionado con la interculturalidad, tuvo un gran auge. Es así como uno de los ejes temáticos fue: " $E d u$ cación superior, diversidad cultural e interculturalidad en América Latina". Además, se reconoce "el rol estratégico de las artes y la cultura en el proceso de producción de conocimientos con compromiso social, en la lucha por la soberanía cultural y la integración pluricultural de las regiones". Asimismo, incluye a las artes como parte de los medios para la libertad y la igualdad, por lo que se han de garantizar sin distinción social, género, etnia, religión ni edad. Esto sin duda, constituye un salto cualitativo en la valoración de las artes y la cultura en el ámbito de la ES. Esto se ve refrendado en el plan de acción propuesto para 2018-2028, donde se plantean metas y estrategias donde las artes están consideradas. Ejemplos de ello son: la estrategia que habla de "desarrollar un programa de reconocimiento científico/artístico para los y las investigadores/as y artistas, de carácter regional..."; la que propone "fomentar matrices de legitimación y evaluación específicas para los procesos de enseñanza y de aprendizaje e investigación en humanidades y artes, en el ámbito de los procesos de generación de conocimientos" y; la que plantea "Crear programas y espacios públicos para el aprendizaje lúdico y disfrute de las ciencias y las artes”. Si bien, podría considerarse que las artes aún no cobran y rol protagónico, es un gran avance.

En suma, tal como lo han expresado grandes pensadores, dentro de los que se cuenta Austin, Nietzsche, Heidegger y Maturana, el lenguaje describe al objeto y constata lo que ya existe. Además, nos permite hablar de ellos, constituyéndose en una de las principales formas de comunicación y por ende facilita la acción. Por tanto, el lenguaje permite construir realidades (ECHEVERRIA, 2003). En consecuencia, resulta interesante reflexionar sobre la ausencia o escasa mención de las artes y letras en los documentos que pretenden dar luces sobre el horizonte hacia el que se debe encaminar la Educación Superior. Aun cuando, esto ha evolucionado positivamente con el correr de los años, no se percibe aún la valoración que debiera tener.
3 La presencia del Arte y la cultura en la Educación Superior

Otro aspecto importante por considerar para aproximarnos a la reflexión que se quiere hacer es lo relativo a los espacios en los cuales está presente el arte y la cultura en las IES y, el rol que cumple en cada uno de ellos. Esto nos permitirá esbozar esa línea imaginaria hacia donde se podría encaminar la internacionalización, teniendo presente el contexto y la concepción de internacionalización que hoy en día se esgrimen.

El arte y la cultura está presente en varios ámbitos de la ES, dentro de los que se cuentan: la formación de profesionales; la enseñanza a través de las artes; las expresiones artísticas y culturales de la comunidad educativa y; la custodia y cuidado del patrimonio cultural.

Considerando que, es probable que existan otros espacios que no hayan sido mencionados en la lista anterior, es innegable presencia de las artes y la cultura en gran parte del quehacer de las IES. Además, en cada uno de los espacios, estas pueden cumplir diversos roles y, en todos ellos se puede visualizar una oportunidad de la internacionalización. Por tanto, a continuación, se revisarán algunas de las posibilidades de incorporar lo internacional, a la vez que se darán algunos ejemplos. Esto ofrecerá un panorama de las actividades posibles de llevar a cabo en post de este objetivo.

\subsection{La formación de profesionales}

Las profesiones relacionadas con las artes y letras, así como también la cultura, cubren un amplio espectro de posibilidades, las cuales podrían ser agrupadas en: disciplinares de expresiones artisticas; profesorado en artes, letras o dimensiones culturales; disciplinas concernientes a las expresiones culturales y; profesiones en temas afines a las artes y la cultura (ver Figura 1).

En todas ellas, el arte y la cultura se articula con el proceso de enseñanza y aprendizaje de una disciplina. Por lo tanto, el arte y la cultura se constituyen en un conocimiento por aprender y para el caso de los docentes, por entregar a sus estudiantes. Además, se ponen en juego saberes que están ligados con la creatividad y con el desarrollo de un pensamiento reflexivo, los cuales pueden aportar a la construcción de la identidad, ciudadana y la formación para la vida (MARDONES, 2008). Por tanto, si bien la internacionalización puede plantearse de manera similar al resto de las carreras, puede alcanzar nuevos matices.

En la línea de lo anterior, se debe tener en cuenta la diversificación de las expresiones del arte, la multi e interculturalidad y la apertura de los espacios de expresión artístico cultural. Esto le imprime nuevos desafíos 

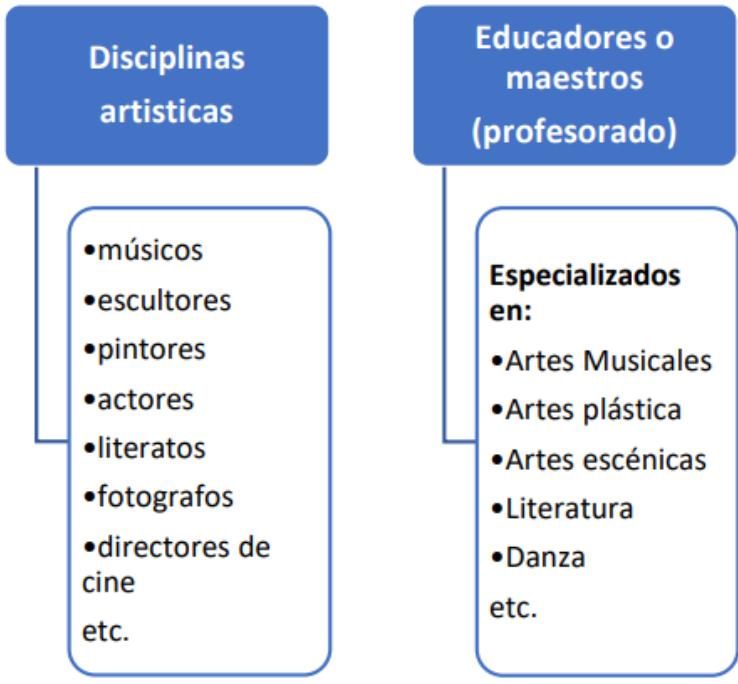

a la formación de profesionales en esta área y por ende también a la forma en que se ha de realizar la internacionalización de estas, tales como: la movilidad estudiantil y docente; la remodelación curricular de pre y post grado que incorporen las nuevas tecnologías, materiales y formas de expresión artístico culturales; la formación de docentes que respondan a las nuevas demandas en cuanto a tipo y forma de enseñanza y aprendizaje, promoviendo la pertinencia y calidad de los contenidos, donde los profesionales puedan desenvolverse más allá de las fronteras, sin que esto signifique la homogenización ni la pérdida de la identidad cultural.

Al mismo tiempo, es necesario tener presente que las industrias culturales, producto de la globalización, han tenido un crecimiento explosivo y por ende se han constituido en una oportunidad para el sostenimiento económicos de los profesionales del área, pero también para fortalecer la identidad de los modelos culturales nacionales, teniendo en cuenta que la producción artístico cultural se ha convertido en un importante actor en la comunicación social y en la constitución del discurso público, el cual se difunde masivamente, a través de los diversos medios de comunicación (CANCLINI, 2000 QUARTESAN; ROMIS; LANZAFAME, 2007).

\subsection{La enseñanza a través de las artes}

Las artes y las expresiones artísticas suelen ser un importante facilitador en el flujo de saberes y comunicación de emociones, generando espacios comunes en ambientes donde se convive con la diversidad. El lenguaje artístico ofrece “... modos únicos y originales de conocimiento, de expresión y de percepción a través

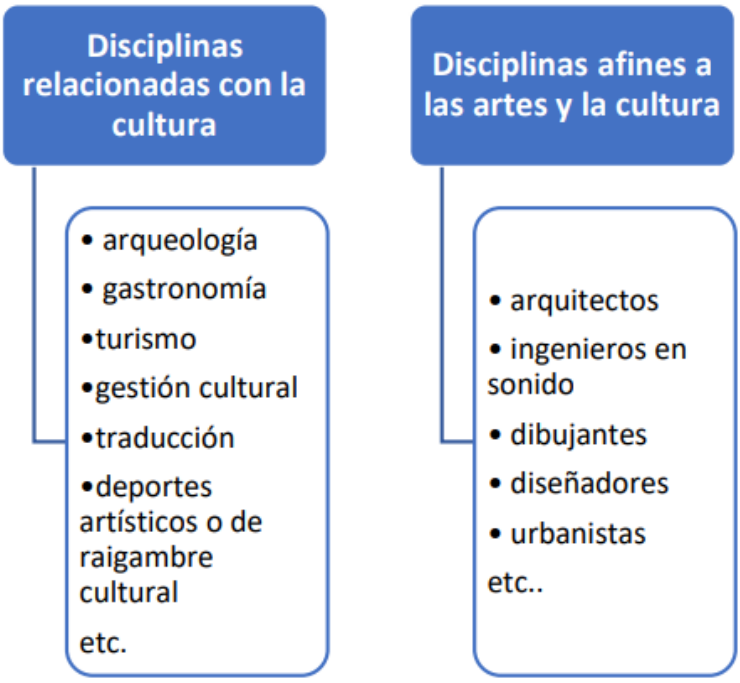

de códigos que les son propios..." (ROS; IANNONE, 2010). Es por lo que se constituye en una importante herramienta pedagógica (MUÑOZ, 2002), la cual es transversal a cualquier disciplina. De igual modo, la incorporación de las artes en los procesos educativo "favorece la motivación frente al estudio y permite desarrollar habilidades transversales a todas las áreas" (OTTONE, 2016).

Por consiguiente, el arte y las expresiones artisticas pueden estar presentes en cualquier área de formación profesional, como por ejemplo el análisis de procesos de enfermedad representados en la pintura, lo cual es un interesante ingrediente para el aprendizaje de profesionales de la salud. O bien, la representación de demandas sanitarias a través de este mismo medio puede entregar elementos de estudio para la salud pública. Con respecto a esto último, se puede mencionar el mural de Diego Rivera en México "La historia de la medicina en México. El pueblo en demanda de salud”, el cual da cuenta de la evolución histórica de la salud y de las tensiones en las demandas de salud de este país (RODRíGUEZ-GóMEZ; CABELLO; RIVERA, 2019).

Del mismo modo, se puede relevar la importancia de un desarrollo integral de los profesionales, cualquiera sea su disciplina y que, por ende, más allá de los contenidos propios y pragmáticos de su quehacer profesional se pueda desarrollar en ellos y ellas la sensibilidad a lo estético-cultural. Es así como se dice que "el desarrollo de la sensibilidad, mediante el crecimiento del sentimiento estético y los imaginarios derivados de las ideas planteadas por los artistas en sus obras" puede ayudar a formar personas más conscientes del mundo que los 
rodea, lo que facilitará la forma de relacionarse con los demás. Así se tendrá a profesionales “... con niveles elevados en valores de orden superior, como la solidaridad, la justicia y la paz.” (FANDIÑO, 2004).

Por consiguiente, la internacionalización en este caso puede verse como una oportunidad de compartir experiencias de enseñanza y aprendizaje a través de las artes. Pero también, es un escenario propicio como para cultivar la inter o multiculturalidad.

\subsection{La expresión artistica y cultural de la comuni- dad educativa}

A la par, se pueden considerar las manifestaciones artísticas como expresión de la cultura de los miembros que componen la comunidad académica (estudiantes, docentes, administradores, directivos, personal de apoyo, etc.). Así es como se pueden observar la realización de actividades artístico-culturales, probablemente en todas las IES. Estas pueden estar ligadas a un estamento en particular (estudiantes, docentes, administrativos o directivos) o en combinación de estos.

Del mismo modo, la cultura y el arte puede convocar la participación de la sociedad civil y la colaboración entre docentes y artistas, artesanos, cultores, lo cual va a promover el enriquecimiento y la vinculación entre comunidades educativas y comunidades locales.

En consecuencia, las artes y la cultura juegan un papel central en el proceso de intercambio que conlleva la internacionalización de la educación superior. Un ejemplo de ello es la experiencia que reporta en una ciudad cubana, donde participaron estudiantes universitarios de 62 paises, quienes realizaron actividades curriculares y extracurriculares en conjunto con la comunidad. La diversidad cultural de los participantes hizo que se generaran estrategias para favorecer la interacción social y el diálogo cultural entre estudiantes, docentes y la comunidad (FERNÁNDEZ et al. 2015).

\subsection{La custodia y el cuidado del patrimonio}

Primeramente, muchas IES son poseedoras de un legado histórico, el cual se materializa en documentos, inmuebles, fotografías, registros fílmicos, colecciones arqueológicas, utensilios dados de baja por el avance tecnológico, artesanías, entre muchos otros. Es misión de las IES ser garantes de este patrimonio, el cual no solo representa el legado de la institución, sino que también de la sociedad a la cual pertenecen dichas instituciones.

De igual manera, las IES deben ser un actor relevante para poner en valor y colaborar en el cuidado del patrimonio cultural de los enclaves geográficos en los cuales se encuentran. Así mismo, la comunidad acadé- mica puede aportar con la generación de conocimiento acerca del del patrimonio propio y el de su entorno social.

Al mismo tiempo, las IES pueden poner a disposición las técnicas e instrumental como para apoyar en la restauración y mantención de los inmuebles patrimoniales o espacios culturales. O también, pueden colaborar en la construcción de los catálogos de obras patrimoniales. De igual forma, podrían hacerse parte de las diligencias tendientes recuperar los bienes culturales sustraídos maliciosamente de sus países de origen.

Lo antes señalado son algunos de los aspectos en los cuales la IES pueden vincularse con la custodia del patrimonio cultural. Todos ellos pueden ser parte de la incorporación de la dimensión internacional de las IES. Una oportunidad, por ejemplo, la brindan los avances tecnológicos y de comunicación, donde se pueden hacer uso de ellos como para poner a disposición de la comunidad nacional e internacional el conocimiento que entregan estos elementos. También, puede considerarse: la generación de museos itinerantes; uso de sistemas de video conferencias para compartir charlas sobre temas relacionadas con el patrimonio, etc.

\section{El horizonte de la internacionalización del Arte y la Cultura en la ES}

El concepto de horizonte, en este caso, hace alusión a su acepción de "límite" y a "un espacio de tiempo" en que se prevé alcanzar un objetivo. Por lo tanto, es un concepto más amplio que lo que podríamos decir de "una meta". Pero si nos remitimos a la cronología del razonamiento, antes de plantearse "un horizonte", es inevitable haber clarificado el porqué y el para qué, para luego delinear el cómo.

\subsection{El porqué de la internacionalización del arte y la cultura en la ES}

En este caso la respuesta es medianamente clara, puesto que, si antes fue considerado relevante, hoy es prácticamente imprescindible. Esto porque en el escenario actual, una IES no puede quedarse enclaustradas en sus muros áulicos. Los estudiantes de hoy, para bien o para mal, viven interconectados, aunque no necesariamente comunicados, con gran parte del planeta. Además, disponen de una cantidad de información incalculable. Los avances de la tecnología, además, han abierto abundantes posibilidades de impartir la ES, así como también han diversificado los materiales y formas de expresión de las artes y la cultura.

Al mismo tiempo, es preciso tener en cuenta que el quehacer de la IES tiene sus raíces en la sociedad y 
en la formación de ciudadanos que puedan transitar por los diferentes escenarios transnacionales, sin perder el rumbo identitario (MONCADA, 2011).

Además, la apertura de la IES a las artes y la cultura puede constituirse en "... un motor de progreso humano y social, y su contraste con la realidad internacional es garantía de estar sustentada en bases firmes para desarrollar correctamente los valores que posee...", además, la internacionalizar la cultura es mucho más que divulgar las actividades de una institución o una nación, sino que da a conocer la caracteristicas de los lugares y habitantes, así como también permite el descubrimiento de nuevas formas de crear, producir o establecer alianza de colaboración (GONZáLEZ 2010).

\subsection{El para qué de la internacionalización del arte y la cultura en la ES}

Aquí, la respuesta no es tan prístina, puesto que dependerá del ámbito de competencia de la ES en el cual se pretenda llevar a cabo la incorporación de una dimensión internacional. Pero también depende del foco que se le quiera dar. $\mathrm{Y}$ en esto último se visualiza que, al menos en el contexto latinoamericanos, se ven tensionadas la visión economista y subyugada a las contingencias del mercado, inspirada en el plan Bolonia, con un enfoque social, reivindicatorio en cuanto a que la ES se ha de considerar como un bien público y social. Además, se destaca el hecho que en la región conviven enormes diferencias sociales (MONCADA, 2011). Por tanto, en lo que a Latinoamérica se refiere, el "para qué" debería ser: para ayudarnos, para potenciarnos, para dar respuesta a los problemas que nos aquejan, para fortalecer nuestra identidad, para formar profesionales conscientes de sus responsabilidades con la sociedad de la que provienen, entre muchas otras.

\subsection{El cómo de la internacionalización del arte y la cultura en la ES}

La respuesta en esta materia es aún más difícil de responder. Para ello deberemos remitirnos a algunos aspectos del contexto que se trataron anteriormente. El primero de ellos tiene que ver con la globalización y el concepto de "aldea global". Pues si pensamos en "el cómo", deberíamos considerar que la internacionalización podría encaminarse hacia la construcción de una "aldea", pero entendida como la raíz originaria del concepto, es decir en un ambiente de mayor intimidad, confianza mutua y solidaridad entre los actores involucrados, respetando las diferencias y esforzarnos por buscar lo que nos une, en vez de los que nos separa.
Por otra parte, en lo relativo al plan Bolonia, sería importante dejar atrás la pretensión de construir espacios comunes de educación superior de la misma forma que se ha hecho en Europa, puesto que, al menos en el contexto latinoamericano, las condiciones sociales y económicas no son emulables. Aun así, es importante rescatar aquellos aspectos que pueden ser buenos de implementar en región, tomando la precauciones en cuanto las condiciones sociopolíticas, económica y de tradición histórica que le son propias a la región (BLANCO et al., 2012).

En lo que respecta al arte y la cultura se ha de tener presente la función social que ella representa, dado que la mutación que ha experimentado la industria cultural ha tendido a desdibujar esta función (SZPILBARG; SAFERSTEIN, 2014).

Ahora bien, sin desconocer la jerarquía que representan la IES del hemisferio norte, en nuestra región también existen IES que brindan educación de pre y post grado con altos estándares de calidad. Por lo tanto, "el cómo" también debiera pensarse con un sentido latinoamericano, donde nuestros estudiantes y académicos puedan intercambiar experiencias y conocimientos que le sean pertinentes a nuestros paises.

\section{Consideraciones finales}

Lo expuesto en este documento, devela una evolución y profundización de los conceptos de internacionalización de la ES, lo cual trae consigo una ventana de oportunidades, tanto para la comunidad educativa como para la sociedad a las que pertenecen dichas instituciones. Sin embargo, es importante tener presente el contexto, el énfasis y los contenidos que se pondrán en juego en este proceso.

La educación superior, históricamente, se ha considerado como una palanca de cambio y de movilidad social, por lo que resulta relevante tener presente que la movilidad de saberes entre las naciones debería ser en un marco de equidad y justicia social.

En este contexto, las artes y la cultura juegan un papel central, puesto suelen ser importantes facilitadores en el flujo de conocimientos y de la comunicación entre los pueblos. Además de propiciar y fortalecer la identidad cultural en espacios de diversidad. Sin embargo, es un área poco explorada y muchas veces subvalorada. Por lo tanto, sería importante llevar a las artes y a la cultura al sitial que le corresponde en los procesos de internacionalización.

En este mismo orden, sería relevante que se reportaran las experiencias, que sin duda existen en gran parte de las IES latinoamericana, puesto que en la medida que se conozcan, se podrán poner en valor, lo cual proba- 
INTERNACIONALIZAÇÃO, EDUCAÇÃO PROFISSIONAL, ARTE E CULTURA: UMA REFLEXÃO.

blemente servirá de agente catalizador e inspirador de nuevos emprendimientos en esta línea.

\section{REFERÊNCIAS}

AGUIRRE, M. E. Los senderos del arte, la formación y la educación artística. notas para un deslinde. Revista Educación y Pedagogía, v. 21, n. 55, p. 15-29, 2009.

ALTBACH, P. G.; KNIGHT, J. Visión panorámica de la internacionalización en la educación superior: motivaciones y realidades. Perfiles educativos, Instituto de Investigaciones sobre la Universidad y la Educación, UNAM, v. 28, n. 112, p. 13-39, 2006.

BENEITONE, P. De la cooperación internacional universitaria a la internacionalización de la educación superior:, cambio de paradigma o maquillaje conceptual. In: (COMP.), G. T. (Ed.). Desde el sur: miradas sobre la internacionalización. Lanus: Ediciones de la UNLa, 2014. p. 187.

BIANCHETTI, L. O processo de bolonha ea submissão de ensino superior à formação para o mercado.

Constelaciones. Revista de Teoría Crítica, v. 6, n. 6, p. 426-435, 2014.

BIANCHETTI, L.; MAGALHÃES, A. M. Declaração de bolonha e internacionalização da educação superior: protagonismo dos reitores e autonomia universitária em questão. Avaliação: Revista da Avaliação da Educação Superior (Campinas), SciELO Brasil, v. 20, n. 1, p. 225-249, 2015.

BLANCO, O.; MARCHITELLI, H.; VARELA, E.; BACCANELLI, M.; SIUFI, G.; TAURO, N. La internacionalización de la educación superior: cómo el instituto universitario gestiona esta nueva función estratégica. Revista del Hospital Italiano, v. 32, n. 1, p. 19-23, 2012.

BOLÍVAR, A. et al. Globalización e identidades:(des) territorialización de la cultura. Revista de educación, v. 1, n. 1, p. 265-288, 2001.

BRUNNER, J. J. El proceso de bolonia en el horizonte latinoamericano: límites y posibilidades. Revista de Educación, Ministerio de Educación y Ciencia, v. 18, n. 1, p. 127-128, 2008.

CANCLINI, N. G. Industrias culturales y globalización: procesos de desarrollo e integración en américa latina. Estudios internacionales, JSTOR, v. 1, n. 1, p. 90-111, 2000.

CONCEPTO. Arte. 2019. Disponível em: $<$ https://concepto.de/arte/>.
CRAVINO, A.; ROLDÁN, J. E. Los desafíos y amenazas de la internacionalización de la educación superior. Revista Electrónica De Didáctica En Educación Superior, v. 1, n. 16, p. 1-16, 2018.

CRES. Conferencia Regional de Educación Superior 1996. 1996. Disponível em: <http: //www.cres2018.org/historia/cres-1996>. Acesso em: 14 mar. 2019.

CRES. Declaraciones y plan de acción. Perfiles Educativos, v. 31, n. 125, p. 90-108. 2009. Disponível em: <http://www.scielo.org.mx/scielo.php?script=sci_ arttext\&>. Acesso em: 07 jul. 2019.

CRES. Declaración CRES 2018. 2018.

Disponível em: <https://drive.google.com/file/d/ 1ipalv-U9v2-kfAY40CEqax9P_9eS8skd/view>.

Acesso em: 31 jul. 2019.

DEHESA, G. d. 1.; KRUGMAN, P. Comprender la globalización. 1. ed. [S.1.]: Alianza, 2007.

ECHEVERRIA, R. Ontología del lenguaje. 1. ed. Santiago: Lom Ediciones S. A, 2003.

EEES. Declaración de Bolonia. 1999. Disponível em: <http://eees.umh.es/contenidos/Documentos/ DeclaracionBolonia.pdf $>$.

ELEKTRART. Diseño de instalaciones

de Arte Digital para eventos. 2019.

Disponível em: <http://elektrart.com/ diseno-de-instalaciones-de-arte-digital-para-eventos/ $>$

FANDIÑO, J. M. El arte y la educación superior.

Educación y educadores, v. 7, n. 1, p. 229-235, 2004.

FANJUL, E. Qué es la globalización. 2018.

Disponível em: <http://www.iberglobal.com/files/ 2018-2/que_es_la_globalizacion(1).pdf>. Acesso em: 11 jul. 2019.

FAZIO, H. V. ¿ Qué es la globalización?: contenido, explicación y representación. 1. ed. Colombia: Ediciones Uniandes-Universidad de los Andes, 2018.

FERNÁNDEZ, N. R.; GARCÍA, E. L.; PÉREZ, M. M.; ALEMÁN, E. B.; OBREGÓN, B. R. P.; LÓPEZ, J. F. La internacionalización de la cultura: contribución a la formación integral de profesionales de la salud. Edumecentro, 1999, Editorial Ciencias Médicas, v. 7, n. 1, p. 76-88, 2015.

FERNÁNDEZ, S. P. La glocalización de la comunicación. Ámbitos. Revista Internacional de Comunicación, v. 1, n. 7-8, p. 151-163, 2002. 
INTERNACIONALIZAÇÃO, EDUCAÇÃO PROFISSIONAL, ARTE E CULTURA: UMA REFLEXÃO.

FIGUEROA, M. d. C. G. Globalización vs multiculturalidad en colombia. EDUCARE ET COMUNICARE: Revista de investigación de la Facultad de Humanidades, v. 3, n. 1, p. 87-91, 2015.

GACEL-ÁVILA, J. Impacto del proceso de bolonia en la educación superior de américa latina. RUSC. Universities and Knowledge Society Journal, Catedra UNESCO de e-learning, v. 8, n. 2, p. 123-134, 2011.

GIANNETTI, C. Estética digital: Sintopía del arte, la ciencia y la tecnología. 1. ed. Barcelona: L'Angelot, 2002.

GONZáLEZ, T. Plan Estratégico para la Internacionalización de las Artes Escénicas en Euskadi. 2010. Barcelona.

GUTIÉRREZ. Continuidades y recuperaciones de la cultura hispana en la construcción de las identidades nacionales americanas. In: GUZMáN, F.; MARTíNEZ, J. M. (Ed.). Arte americano e Independencia. Nuevas iconografías. Santiago: Universidad Adolfo Ibáñez, 2010. p. 29-40.

HRISTOVA, S.; PETROVSKA, I.; DIMITROVA, M. INTERNATIONALIZATION IN HIGHER EDUCATION: TRENDS AND OPPORTUNITIES.

2013. Analele Universitatii'Eftimie Murgu'Resita. Fascicola II. Studii Economice.

KAUFMANN, S. Multiculturalidad y ética del reconocimiento. Persona y sociedad, v. 25, n. 1, p. 75-87, 2011.

KNIGHT, J. Internationalization: Elements and Checkpoints. CBIE Research No. 7. 1. ed. Ottawa: ERIC, 1994.

Las Heras, J. El grito de Córdoba: la Reforma Universitaria de 1918 y su vigencia en la universidad del siglo XXI. 1. ed. Santiago: Editorial Universitaria, 2009.

LLULL, J. P. Evolución del concepto y de la significación social del patrimonio cultural. Arte, individuo y sociedad, Universidad Complutense de Madrid, v. 17, p. 177-206, 2005.

MARDONES. Buenos Aires. 2008.

MARÍN, E. H. El retorno de la diáspora artística latinoamericana/la acción diferida del desplazamiento. Cadernos de Estudos Latino-Americanos, v. 3, n. 1, p. 85-125, 2007.
MECA, J. Patrimonio cultural:¿ espacio de imaginación o de especulación. In: CARREñO, C. (Ed.). Cuadernos de patrimonio cultural, Reflexiones contemporáneas. Tunja: Universidad Pedagógica y Tecnológica de Colombia, 2016.

MONCADA, J. S. C. Hacia una internacionalización solidaria de la educación superior. Theoria,

Universidad del Bío Bío, v. 20, n. 1, p. 21-32, 2011.

MUÑOZ, M. A. Trayectos de las artes plásticas en la argentina del siglo xx. Inti, JSTOR, v. 1, n. 52, p. 511-533, 2000.

MUÑOZ, M. P. La educación a través del arte en la educación social: Los espacios laborales y la investigación en educación a través del arte. Pedagogía social: revista interuniversitaria, Sociedad Iberoamericana de Pedagogía Social, v. 1, n. 9, p. 287-299, 2002.

NILAN, P.; FEIXA, C. \& Una juventud global? Identidades híbridas, mundos plurales (ponencia). 1. ed. Instituto de Comunicación e Imagen de la Universidad de Chile, 2010

Olímpio Bento, J. Proceso de bolonia: una ofensa y traición a la idea y misión. In: MERCADO

DE LETRAS. EL PROCESO DE BOLONIA Y LA GLOBALIZACIÓN DE LA EDUCACIÓN SUPERIOR. Antecedentes, implementación y repercusiones en el quehacer de los trabajadores de la educación. San Pablo: Consejo Latinoamericano de Ciencias Sociales, 2016.

OTTONE, E. Caja de Herramientas para la Educación Artística. [S.1.]: Recuperado de http://www. cultura. gob. cl/wp-content/uploads/2016/02, 2016. Consejo Nacional De La Cultura Y Las Arte. Enseñanza y Aprendizaje Artístico.

QUARTESAN, A.; ROMIS, M.; LANZAFAME, F. Las industrias culturales en América Latina y el Caribe: desafíos y oportunidades. 2007. Washington DC: Banco Interamericano de Desarrollo.

Real Academia Española. Diccionario de la lengua española. 2018. Disponível em: <http://www.rae.es/> Acesso em: 29 nov. 2013.

REDACCIóN. Tecnología y arte: ¿Qué sucede cuando se fusionan? 2018. Disponível em: $<$ https://rpp.pe/campanas/contenido-patrocinado/ tecnologia-y-arte-que-sucede-cuando-se-fusionan\ -noticia-1162011> Acesso em: 7 abr. 2019. 
RODRíGUEZ-GóMEZ, G.; CABELLO, F. C.;

RIVERA, D. La historia de la medicina en méxico. el pueblo en demanda de salud, mural de 1953 aún vigente. Revista Chilena de Pediatría, v. 90, n. 3, p. 351-355, 2019.

ROS, N.; IANNONE, N. Formación y capacitación docente en educación artística: Dos propuestas pensadas desde el profesorado y licenciatura en educación inicial para trabajar los nuevos escenarios educativos. Revista Iberoamericana de Educación, v. 52, n. 2, p. 1-10, 2010.

SOLIS, E. L. Corea del Sur como ejemplo del fracaso de la cultura global. La hibridacion cultural como respuesta a la occidentalizacion. Tese (Tesis Licenciatura. Relaciones Internacionales) Universidad de las Américas Puebla, Cholula, Puebla, 2005.

SZPILBARG, D.; SAFERSTEIN, E. A. De la industria cultural a las industrias creativas: un análisis de la transformación del término y sus usos contemporáneos. Estudios de filosofía práctica e historia de las ideas, v. 16, n. 2, p. 99-112, 2014.

TELLO, C. Políticas educativas, educación superior y proceso de bolonia en latinoamérica. Crítica Educativa, v. 1, n. 1, p. 80-97, 2015.

UNESCO. Universal Declaration on Cultural

Diversit. Paris: [s.n.], 2001. Disponível em: <http://portal.unesco.org/en/ev.php-URL_ID $=13179 \&$ URL_DO=DO_TOPIC\&URL_SECTION=201.html>

UNESCO. Declaracion de bogota sobre educacion artistica. In: Conferencia regional de américa latina y el caribe sobre educación artística. Bogotá: Unesco, 2005. p. 1-3.

UNESCO. Hoja de ruta para la educación artística. In: Conferencia mundial sobre la educación artística: Construir capacidades creativas para el siglo XXI. Lisboa: Unesco, 2006.

UNESCO. World Heritage. 2019. Disponível em: $<$ http://whc.unesco.org/en/about/>

UNIVERSIDAD NACIONAL DE LAS ARTES.

Historia. 2019. Disponível em: <https: //visuales.una.edu.ar/contenidos/historia_12468>

WIT, H.; GACEL-ÁVILA, J.; KNOBEL, M. Estado del arte de la internacionalización de la educación superior en américa latina. Revista de Educación Superior en América Latina, v. 1, n. 2, p. 2-5, 2017. 